\title{
Estudio comparativo del currículo de matemáticas ofertado en ingenierías por la Universidad Distrital Francisco José de Caldas*
}

\author{
Comparative study of the mathematics curriculum offered in engi- \\ neering from the University Distrital Francisco José de Caldas \\ Estudo comparativo do currículo de matemática oferecido em en- \\ genharia pela Universidade Francisco José de Caldas
}

Edier H. Bustos**

Edilberto Sarmiento***

Esperanza Flórez ${ }^{* * * *}$

\section{Resumen}

Este documento es producto de una investigación cualitativa, básica por su finalidad, pero aplicada, ya que soporta posibles reformas y ajustes curriculares en el área de matemáticas; también permitirá determinar el estado actual del currículo de matemáticas de la Universidad Distrital de universidades nacionales, entre las que se encuentran, la Universidad Distrital Francisco José de Caldas, Universidad del Valle, Universidad Industrial de Santander, Universidad Nacional de Colombia, Universidad del Valle, Universidad de Antioquia, Universidad del Tolima, Universidad Piloto y Universidad de los Andes.

El lector podrá encontrar que muchos de los espacios académicos de matemáticas, ofertados por la Universidad Distrital, son los mismos que ofrecen otras instituciones universitarias; pues, a pesar de la polisemia de nombres referidos a las mismas unidades temáticas y la relación equivalente en créditos e intensidad horaria, la única diferencia son las asignaturas que se ofertan en primer semestre, especialmente porque algunas de las IES ofertan précalculo antes de cálculo diferencial.

Palabras clave: currículo de matemáticas, investigación curricular.

\section{Abastract}

This paper arises as a result of an investigation, which is characterized by qualitative, basic and applied their purpose in that final products will support curriculum reforms and adjustments possible in the area of

Proyecto de investigación financiado por la Universidad Distrital Francisco José de Caldas, titulado "Estado del currículo de matemáticas de los programas de Ingenierías y Tecnologías, ofertados por la Universidad Distrital Francisco José de Caldas.

** Magíster en Docencia de las Matemáticas y Docente de la Universidad Distrital Francisco José de Caldas. Correo electrónico: ehbustosv@udistrital.edu.co

**** Licenciado y Magíster en Matemáticas, Docente de la Universidad Distrital Francisco José de Caldas. Correo electrónico: esarmiento@udistrital.edu.co

***** Magíster en Docencia de las Matemáticas y Docente de la Universidad Distrital Francisco José de Caldas. Correo electrónico: espflorez@yahoo.com 
mathematics. Possible to determine the current status of the mathematics curriculum of the University Francisco José de Caldas District; This has been studied in universities around the National Curriculum. These include the District University Francisco José de Caldas, Universidad del Valle, Universidad Industrial de Santander, Universidad Nacional de Colombia, Universidad del Valle, Universidad de Antioquia, Uniandes, Universidad del Tolima, Universidad Piloto.

The reader will find that many of the academic areas of math offered in the University District, are the same as those offered in other universities in spite of the many meanings of names referring to the same thematic units, with a ratio equivalent in credits and intensity time, with differences in the courses on offer in the first half, especially because some of IES offering pre-calculus precalculus prior to differential calculus.

Keywords: math curriculum, curriculum Research

\section{Resumo}

Este trabalho é resultado de uma investigação, que se caracteriza por qualitativa básica e aplicada para o fim de que os produtos finais, os eventuais reformas curriculares e ajustes na área da matemática. Permitindo determinar o estado atual do currículo de matemática da Universidade Francisco José de Caldas, por isso foi estudado em universidades de todo o currículo nacional, entre elas estão a Universidade Francisco José de Caldas, Universidad del Valle, Universidad Industrial Santander, Universidad Nacional de Colombia, Universidad del Valle, Universidad de Antioquia, Tolima University, Universidad Piloto, Universidad de los Andes.

O leitor vai descobrir que muitas das áreas acadêmicas de matemática oferecidos no Distrito Universidade, são os mesmos que os oferecidos em outras universidades, apesar da polissemia de denominações para as mesmas unidades temáticas, com um rácio de créditos equivalentes e intensidade vezes, com diferenças nas disciplinas que são oferecidas no primeiro semestre, especialmente porque algumas das IES que oferecem pré cálculo precalculus

Palavras-chave: matemática currículo, pesquisa currículo

\section{Introducción}

Este artículo responde, de alguna manera, al estado actual de la educación y los requerimientos de la sociedad "haciendo referencia a una sociedad donde el conocimiento es un factor fundamental del progreso material y cultural" (Palacio, 2004). En este sentido, la flexibilidad curricular es un factor primordial para la adquisición de nuevos conocimientos, puesto que permite a los estudiantes moverse dinámicamente a través del currículo, según sus intenciones y finalidades; además los ayuda, junto con los docentes a "generar conocimiento acerca de su realidad y de su entorno [siendo] capaces de utilizar dicho conocimiento para concebir y construir su futuro. De esta forma, el conocimiento se convierte no sólo en instrumento para explicar y comprender la realidad, sino también en motor de desarrollo y factor dinamizador del cambio social" (Chaparro, 1999: 252). 
En las últimas décadas los currículos de matemáticas han cumplido un factor dinamizador en la conformación de un nuevo sistema educativo, basado en las ciencias de la tecnología y la electrónica. También han logrado permear, con su acción e innovación, todas las ramas de la producción industrial que necesariamente requieren de la utilización de nuevas tecnologías, en donde los estudios a nivel curricular deben ir acompañados de

[...] cambios de competencias [que] conciernen tanto a los obreros directos como a los técnicos, los tecnólogos y los ingenieros. Particularmente estos últimos y los cuadros de dirección han visto radicalmente modificados el tipo de trabajo que deben llevar a cabo y las competencias necesarias para poder cumplir sus labores. La formación de estas competencias precisa cambios profundos en la educación superior. (Misas Arango, 2004).

Bajo esta perspectiva, este trabajo confronta, semestre a semestre, los espacios curriculares del área de matemáticas con los espacios curriculares de la misma área en universidades nacionales.

En este orden de ideas, la investigación se desarrolló en dos fases: la primera se propuso como objetivo diseñar un instrumento (matriz) que permitiera organizar la recolección de la información y facilitara el posterior análisis, referente a la cantidad de espacios académicos matemáticos distribuidos en la malla curricular, objetivos, contenidos, intensidad horaria y créditos.

La investigación se desarrolló, inicialmente, recopilando información documental sobre el currículo de matemáticas, producida en las instituciones de educación superior que ofertan ingenierías y tecnologías, relacionados con las carreras que ofrece la Universidad Distrital Francisco José de Caldas. Esta compilación de información documental se orientó por la definición de los aspectos curriculares que se consideraron de fundamental importancia para el estudio comparativo y que abarcarán todo tipo de fuentes documentales relacionadas con el objetivo propuesto.

En la segunda fase, relacionada con el carácter cualitativo de esta investigación, se asumió el currículo como una construcción social en la que participan actores de la práctica académica, tomando como punto de partida sus experiencias, subjetividades particulares, concepciones y definiciones. A partir de las interpretaciones y los juicios de valoración, se caracterizó la realidad curricular en matemáticas de los programas de Ingeniería y tecnología de la Universidad Distrital Francisco José de Caldas.

Por lo tanto, las interpretaciones y los juicios de valoración que se hagan son el resultado de metodologías distintas a las usadas en la investigación cuantitativa. Aquí se busca caracterizar la realidad curricular de los distintos programas de Ingeniería y Tecnología de la Universidad Distrital Francisco José de Caldas, mediante la elaboración, rigurosa y profunda, del estudio comparativo fijado como objetivo.

\section{Análisis de las universidades nacionales}

Cuando se analizan los diferentes espacios académicos del área de matemáticas en las universidades colombianas y a nivel internacional, se encuentra que las diferencias internas se manifiestan en términos de las asignaturas que se ofertan para cada proyecto curricular o programa.

\section{Primer semestre}

Lo que para la Universidad Distrital Francisco José de Caldas se denomina Cálculo Diferencial, en otras universidades recibe el nombre de Cálculo I, Introducción al Análisis Matemático. Otro espacio académico que se aprecia en las instituciones es el denominado Precálculo, Fundamentos de Matemática I o Matemáticas Básicas (ver Tabla 1).

\begin{tabular}{|c|c|c|c|}
\hline $\begin{array}{c}\text { Asignatura } \\
\text { de primer } \\
\text { semestre }\end{array}$ & $\begin{array}{c}\text { Cantidad } \\
\text { de univer- } \\
\text { sidades }\end{array}$ & $\begin{array}{c}\text { Intensidad } \\
\text { horaria } \\
\text { (semanal) }\end{array}$ & Créditos \\
\hline $\begin{array}{c}\text { Cálculo } \\
\text { Diferencial }\end{array}$ & $76,5 \%$ & 4,506 & 4 \\
\hline Precálculo & $23,5 \%$ & 304 & 304 \\
\hline
\end{tabular}

Tabla 1 Espacio académico vs. Porcentaje de universidades (primer semestre)

Fuente: elaboración porpia 


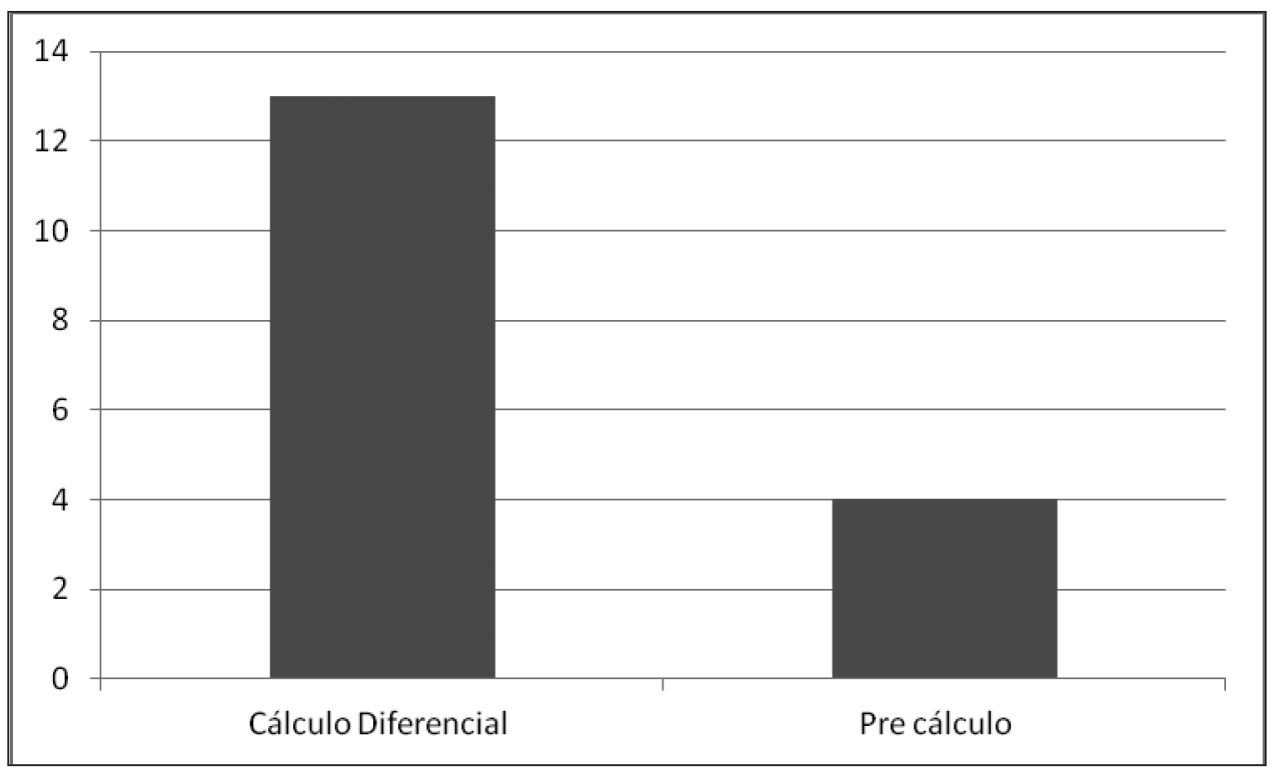

Gráfica 1. Espacio académico vs. Cantidad de universidades (primer semestre)

Fuente: elaboración propia

Vale la pena señalar que ninguno de los programas ofertados por las universidades, ve más de un espacio académico de matemáticas para el primer semestre. En lo referente a los créditos académicos, encontramos que estos oscilan entre 3 y 4 , dependiendo de la intensidad horaria.

Es de resaltar, el interés que tienen algunas universidades por fundamentar los preconceptos de matemáticas, que deben tener los estudiantes antes de enfrentarse a los cálculos. En virtud de lo cual, abordan conceptos como los siguientes: Funciones, Teoría de los números reales, Polinomios y Teoría de Ecuaciones, Sistema de Ecuaciones, Inecuaciones, Conceptos Básicos de la Geometría Euclidiana, y Conceptos Básicos de la Geometría Analítica.

\section{Segundo semestre}

En este semestre encontramos un espacio académico denominado, en la Universidad Distrital Francisco José de Caldas, Cálculo Integral, que en otras universidades se denomina Matemáticas II o Cálculo II. El contenido es el mismo, lo que varía es la intensidad horaria y el número de créditos. También se puede anotar que mientras para unas carreras de ingeniería aparecía esta asignatura del área de matemáticas, en otras aparecía como Cálculo Diferencial e Integral. Asimismo, en este semestre algunas universidades permiten que los estudiantes cursen dos espacios académicos del área de matemáticas (por ejemplo, Cálculo Integral y Álgebra Lineal), dos espacios separados y con asignación horaria para trabajo directo. En este sentido encontramos lo siguiente:

\begin{tabular}{|l|l|l|l|}
\hline $\begin{array}{l}\text { Asignatura } \\
\text { de segundo } \\
\text { semestre }\end{array}$ & $\begin{array}{l}\text { Cantidad de } \\
\text { universida- } \\
\text { des }\end{array}$ & $\begin{array}{l}\text { Intensidad } \\
\text { horaria } \\
\text { (semanal) }\end{array}$ & $\begin{array}{l}\text { Número } \\
\text { de } \\
\text { créditos }\end{array}$ \\
\hline $\begin{array}{l}\text { Algebra } \\
\text { Lineal }\end{array}$ & $82,4 \%$ & 4 & 4 \\
\hline $\begin{array}{l}\text { Cálculo } \\
\text { Integral }\end{array}$ & $70,6 \%$ & 3,406 & 304 \\
\hline $\begin{array}{l}\text { Cálculo } \\
\text { Diferencial e } \\
\text { Integral }\end{array}$ & $11,8 \%$ & 6 & 4 \\
\hline $\begin{array}{l}\text { Cálculo } \\
\text { Integral con } \\
\text { Ecuaciones } \\
\text { Diferenciales }\end{array}$ & $5,9 \%$ & 6 & 4 \\
\hline $\begin{array}{l}\text { Cálculo } \\
\text { Diferencial }\end{array}$ & $11,8 \%$ & 406 & 4 \\
\hline
\end{tabular}

Tabla 2. Espacio académico vs. Porcentaje de universidades (segundo semestre)

Fuente: elaboración propia 


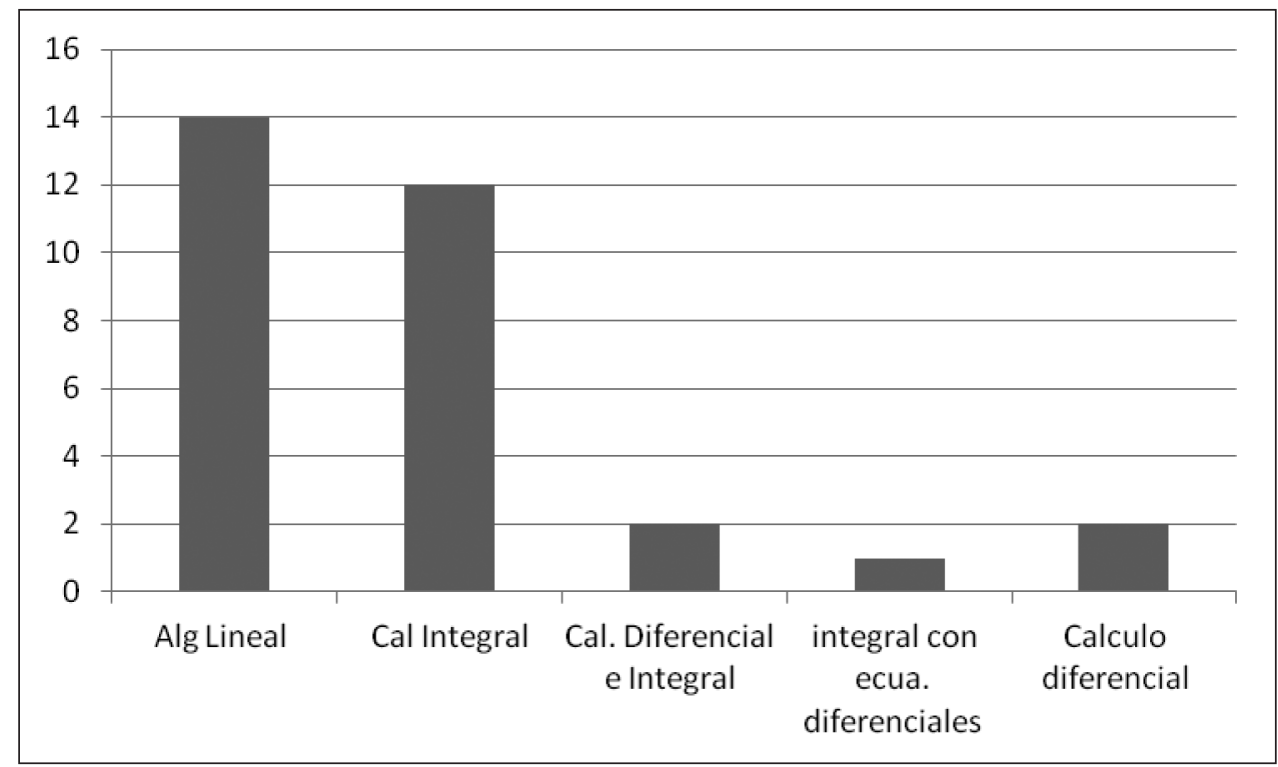

Gráfica 2. Espacio académico vs. Cantidad de universidades (segundo semestre)

Fuente: elaboración propia

En este semestre encontramos que, de los 17 programas analizados, el 82,4 \% cursan algebra lineal, el $11,7 \%$ cálculo diferencial e integral como un solo espacio académico, el 70,6\% calculo integral, el $11,7 \%$ calculo diferencial y el 5,9\% cursan un solo espacio académico, denominado cálculo integral con ecuaciones diferenciales.

Otro caso es el de la asignación de horas de trabajo directo con los estudiantes. Así, por ejemplo, el $82,4 \%$ deben tener una asignación semanal de matemáticas, que comprenda los espacios académicos de algebra lineal y cálculo integral o algebra lineal y cálculo diferencial, con una duración de entre 8 y 10 horas. Los créditos también estarán supeditados al número de horas por espacio académico. Lo que permite evidenciar la idea de orientar a los estudiantes frente a los conceptos que deben saber para abordar los siguientes cálculos: vectorial o multivariado.

Por otro lado, los distintos y variados criterios de evaluación son evidentes, estos varían de acuerdo a la diversidad de políticas institucionales de las universidades, y están en consonancia con la libertad de cátedra.

\section{Tercer semestre}

Los cálculos ofertados por las universidades en este semestre reciben el nombre de cálculo multivariado (matemáticas III, cálculo III), cálculo vectorial y cálculo integral, también se ofertan algebra lineal y probabilidad y estadística. La diferencia, como se puede evidenciar en términos de las pretensiones entre el cálculo multivariado y el cálculo vectorial, radica en que en el primero se pretende estudiar funciones de varias variables, derivadas direccionales, derivadas parciales e integrales múltiples y algunos aspectos geométricos relacionadas con estas. Mientras que el segundo pretende realizar un análisis real multivariable de vectores en 2 o más dimensiones; de tal forma que se puedan estudiar sistemas de coordenadas, campos escalares y vectoriales, para terminar con el Teorema de Divergencia y Teorema de Stokes. 


\begin{tabular}{|l|c|c|c|}
\hline $\begin{array}{l}\text { Asignatura de } \\
\text { tercer semestre }\end{array}$ & $\begin{array}{c}\text { Cantidad de } \\
\text { universidades }\end{array}$ & $\begin{array}{c}\text { Intensidad hora- } \\
\text { ria (semanal) }\end{array}$ & $\begin{array}{c}\text { Número de } \\
\text { créditos }\end{array}$ \\
\hline Algebra Lineal & $17,6 \%$ & 4 & 4 \\
\hline Cálculo Integral & $11,8 \%$ & 406 & 4 \\
\hline $\begin{array}{l}\text { Cálculo } \\
\text { Multivariado }\end{array}$ & $17,6 \%$ & 4 & 4 \\
\hline Cálculo Vectorial & $23,5 \%$ & 4 & 4 \\
\hline $\begin{array}{l}\text { Estadística y } \\
\text { Probabilidad }\end{array}$ & $47 \%$ & 4 & 4 \\
\hline
\end{tabular}

Tabla 3. Espacio académico vs. Porcentaje de universidades (tercer semestre)

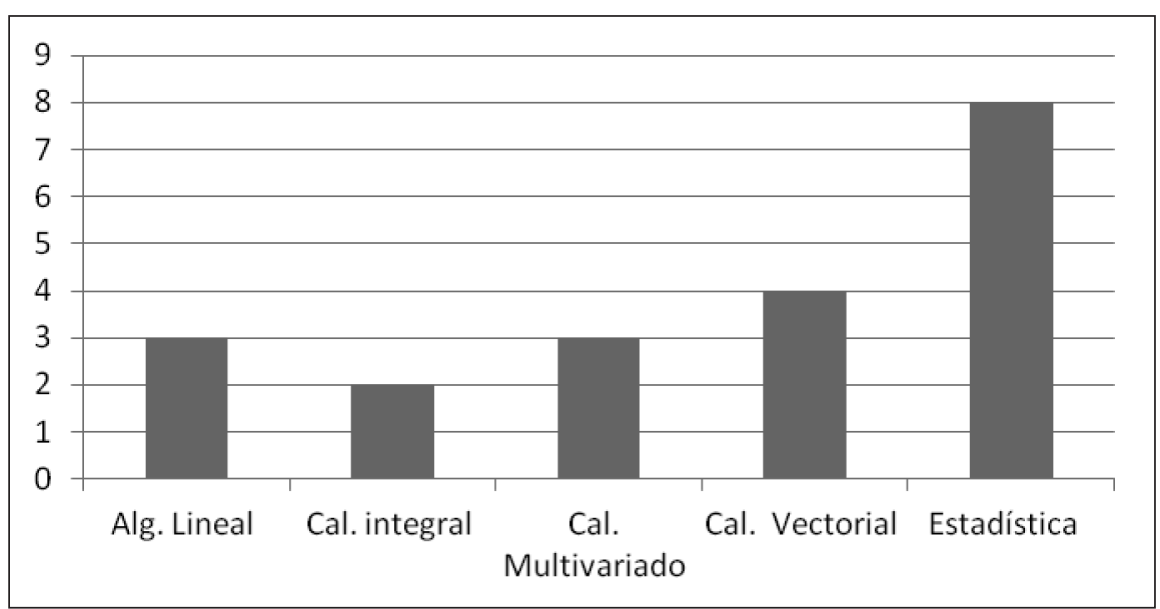

Gráfica 3. Espacio académico vs. Cantidad de universidades (tercer semestre)

Fuente: elaboración propia

Es interesante observar que en este semestre los estudiantes deben dedicarle a las matemáticas entre 8 y 12 horas de trabajo con el docente, ya que el 52,9\% de ellos deben ver más de una asignatura.

\begin{tabular}{|l|c|}
\hline Espacios Académicos & Cantidad de universidades \\
\hline $\begin{array}{l}\text { Cálculo Integral } \\
\text { Algebra Lineal } \\
\text { Estadística y Probabilidad }\end{array}$ & 2 \\
\hline $\begin{array}{l}\text { Cálculo Multivariado } \\
\text { Estadística y Probabilidad }\end{array}$ & 2 \\
\hline $\begin{array}{l}\text { Cálculo Vectorial } \\
\text { Estadística y Probabilidad }\end{array}$ & 4 \\
\hline $\begin{array}{l}\text { Cálculo Multivariado } \\
\text { Algebra Lineal }\end{array}$ & 1 \\
\hline
\end{tabular}

Tabla 4. Más de un espacio académico vs. Número

Fuente: elaboración propia

\section{Cuarto semestre}

En este semestre, algunas universidades continúan con la línea de cálculo, ofertando cálculo vectorial y cálculo multivariado; otras, en cambio, ofrecen ecuaciones diferenciales y probabilidad y estadística, ambas como espacios diferentes. En este sentido, encontramos en, términos generales, lo siguiente: 


\begin{tabular}{|c|c|c|c|}
\hline $\begin{array}{c}\text { Asignatura de cuarto } \\
\text { semestre }\end{array}$ & $\begin{array}{c}\text { Cantidad de } \\
\text { universidades }\end{array}$ & $\begin{array}{c}\text { Intensidad horaria } \\
\text { (semanal) }\end{array}$ & $\begin{array}{c}\text { Número de } \\
\text { créditos }\end{array}$ \\
\hline Cálculo Integral & $5,9 \%$ & 4 & 4 \\
\hline Cálculo Multivariado & $23,5 \%$ & 304 & 4 \\
\hline Cálculo Vectorial & $35,3 \%$ & 4 & 4 \\
\hline Estadística y Probabilidad & $41,2 \%$ & 4 & 4 \\
\hline Ecuaciones Diferenciales & $35,3 \%$ & 4 & 4 \\
\hline
\end{tabular}

Tabla 5. Espacio académico vs. Porcentaje de universidades (cuarto semestre)

Fuente: elaboración propia

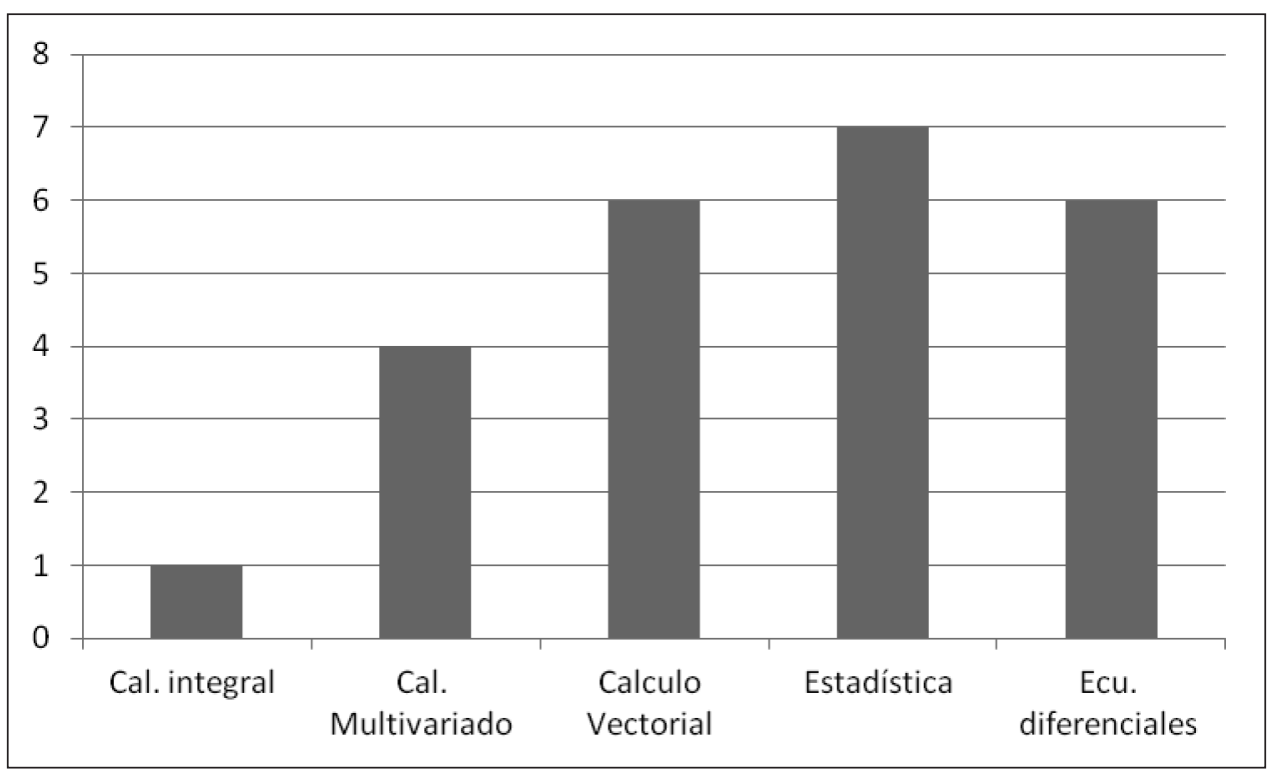

Gráfica 4. Espacio académico vs. Cantidad de universidades (cuarto semestre)

Fuente: elaboración propia

Los estudiantes que cursan más de una asignatura deben tener una dedicación semanal al trabajo directo de 8 horas, relacionadas con un espacio académico del área de las matemáticas y complementada con una asignatura del área de la estadística (ver Tabla 6).

\begin{tabular}{|l|c|}
\hline Espacios Académicos & Cantidad de universidades \\
\hline $\begin{array}{l}\text { Cálculo Multivariado } \\
\text { Estadística y Probabilidad }\end{array}$ & 2 \\
\hline $\begin{array}{l}\text { Cálculo Vectorial } \\
\text { Estadística y Probabilidad }\end{array}$ & 3 \\
\hline $\begin{array}{l}\text { Ecuaciones Diferenciales } \\
\text { Estadística y Probabilidad }\end{array}$ & 2 \\
\hline
\end{tabular}

Tabla 6. Más de un espacio académico vs número de universidades (cuarto semestre)

Fuente: elaboración propia

\section{Quinto semestre}

En el quinto semestre se acaba la línea de las matemáticas. Aquí encontramos que algunas universidades culminan con matemáticas especiales, métodos numéricos o ecuaciones diferenciales; relacionándolas directamente con los tópicos trabajados en los semestres anteriores y tratando de guardar una correspondencia entre cada uno de los temas abordados. De esta forma se organizan los preconceptos necesarios para construir el análisis y estudio de nuevos conceptos matemáticos. 


\begin{tabular}{|l|c|c|c|}
\hline Asignatura & $\begin{array}{c}\text { Cantidad } \\
\text { de univer- } \\
\text { sidades }\end{array}$ & $\begin{array}{c}\text { Intensidad } \\
\text { horaria } \\
\text { (semanal) }\end{array}$ & $\begin{array}{c}\text { Número } \\
\text { de créditos }\end{array}$ \\
\hline $\begin{array}{l}\text { Matemáticas } \\
\text { Especiales }\end{array}$ & $11,8 \%$ & 304 & 4 \\
\hline $\begin{array}{l}\text { Métodos } \\
\text { Numéricos }\end{array}$ & $11,8 \%$ & 4 & 4 \\
\hline $\begin{array}{l}\text { Estadística y } \\
\text { Probabilidad }\end{array}$ & $11,8 \%$ & 4 & 4 \\
\hline $\begin{array}{l}\text { Ecuaciones } \\
\text { Diferenciales }\end{array}$ & $29,5 \%$ & 4 & 4 \\
\hline
\end{tabular}

Tabla 71. Espacio académico vs. Porcentaje de universidades (quinto semestre)

Fuente: elaboración propia

En este semestre es interesante observar que no todas las universidades ofertan cursos de matemáticas, estos dependen en gran medida de las intenciones que tengan cada una de las carreras de

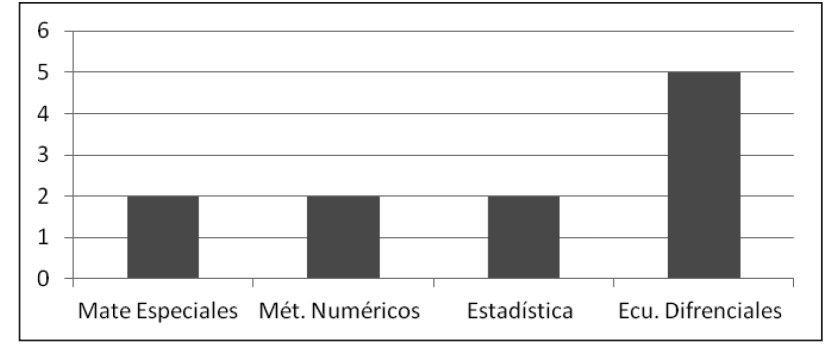

Gráfica 5. Espacio académico vs. Cantidad de universidades (quinto semestre)

Fuente: elaboración propia ingeniería o tecnología. En tal sentido, dentro de las 17 universidades analizadas, tan solo 9 continúan lo que pudiéramos denominar una línea de matemáticas hasta este semestre.

\section{Universidad Distrital Francisco José de Caldas}

\begin{tabular}{|l|c|c|c|}
\hline Espacios académicos & Semestre & $\begin{array}{c}\text { Intensidad trabajo directo } \\
\text { compartido }\end{array}$ & Créditos \\
\hline Cálculo Diferencial & Primero & $6 \mathrm{~h}$ & 4 \\
\hline Cálculo Integral & Segundo & $6 \mathrm{~h}$ & 3 \\
\hline Algebra Lineal & Segundo & $4 \mathrm{~h}$ & 3 \\
\hline Cálculo Multivariado & Tercer & $6 \mathrm{~h}$ & 4 \\
\hline Ecuaciones Diferenciales & Cuarto & $4 \mathrm{~h}$ & 3 \\
\hline Matemáticas Especiales I & Quinto & $6 \mathrm{~h}$ & 4 \\
\hline Matemáticas Especiales II & Quinto & $6 \mathrm{~h}$ & 4 \\
\hline Métodos Numéricos & Quinto & 4 & 4 \\
\hline Probabilidad y Estadística & Tercero & 4 & 4 \\
\hline
\end{tabular}

Tabla 8. Espacios curriculares de matemáticas UDFJC

Fuente: elaboración propia

En los cursos ofertados por la Universidad Distrital Francisco José de Caldas, se puede evidenciar, a manera de ejemplo, que en el desarrollo del cálculo diferencial los estudiantes tienen 6 horas semanales de trabajo directo. Igualmente, en primer semestre tienen que dedicarle (en horas semanales) al estudio de las matemáticas 4 horas de trabajo directo, 2 
horas de trabajo colaborativo y 8 horas de trabajo autónomo (ver Tabla 9).

La Universidad Distrital Francisco "José de Caldas", tiene diferentes formas de trabajo: 1) Trabajo Presencial Directo (TD). Trabajo de aula con plenaria de todos los estudiantes. 2) Trabajo Mediado Cooperativo (TC). Trabajo de tutoría del docente a pequeños grupos o de forma individual a los estudiantes y 3) Trabajo Autónomo (TA). Trabajo del estudiante sin presencia del docente, que se puede realizar en distintas instancias: en grupos de trabajo o en forma individual, en casa, biblioteca o laboratorio, etc. (Ver anexo 3).

\begin{tabular}{|l|c|c|c|c|c|}
\hline Asignatura & Semestre & TD & TC & TA & $\begin{array}{c}\text { Total } \\
\text { horas }\end{array}$ \\
\hline $\begin{array}{l}\text { Cálculo } \\
\text { Diferencial }\end{array}$ & Primero & 4 & 2 & 6 & 12 \\
\hline $\begin{array}{l}\text { Cálculo } \\
\text { Integral }\end{array}$ & Segundo & 4 & 2 & 6 & 12 \\
\hline Algebra Lineal & Segundo & 4 & 2 & 6 & 12 \\
\hline $\begin{array}{l}\text { Cálculo } \\
\text { Multivariado }\end{array}$ & Tercero & 4 & 2 & 8 & 12 \\
\hline $\begin{array}{l}\text { Ecuaciones } \\
\text { Diferenciales I }\end{array}$ & Cuarto & 3 & 1 & 6 & 10 \\
\hline $\begin{array}{l}\text { Ecuaciones } \\
\text { Diferenciales II }\end{array}$ & Cuarto & 3 & 1 & 6 & 10 \\
\hline $\begin{array}{l}\text { Matemáticas } \\
\text { Especiales }\end{array}$ & Quinto & 4 & 2 & 6 & 12 \\
\hline $\begin{array}{l}\text { Métodos } \\
\text { Numéricos }\end{array}$ & Quinto & 4 & 2 & 6 & 12 \\
\hline $\begin{array}{l}\text { Probabilidad y } \\
\text { Estadística }\end{array}$ & Tercero & 4 & 2 & 6 & 12 \\
\hline
\end{tabular}

Tabla 9. Distribución horaria para las asignaturas de matemáticas (UDFJC)

Fuente: elaboración propia

Por otro lado, durante aproximadamente 5 semestres, los estudiantes han estado reflexionando y estudiando los conceptos matemáticos propios de su carrera, lo que podría ser suficiente para lograr que puedan llegar a cumplir con los objetivos que se pretenden.
En relación con el currículo de matemáticas, plasmado en los syllabus (anexo 2) la universidad Distrital Francisco José de Caldas, oferta los conocimientos mínimos en matemáticas que se exigen en las universidades nacionales e internacionales. En tal sentido, los estudiantes de Ingeniería o Tecnología, que deseen homologar los espacios académicos con otra universidad, lo podrán hacer sin ningún inconveniente ya que estos satisfacen en número de créditos, intensidad horaria y contenidos programáticos los de otras instituciones.

Los estudiantes, al terminar la línea de matemáticas, tanto a nivel internacional como a nivel nacional, incluyendo a la Universidad Distrital Francisco José de Caldas deberán haber desarrollado y fortalecido los siguientes aspectos:

- Un pensamiento de tipo abstracto. Deberán estar en capacidad de plantear, resolver e interpretar, de manera crítica, diferentes problemas en los que se utiliza la matemática como herramienta formadora de estructura de pensamiento en este tipo de aplicaciones.

- Capacidad para desarrollar cualquier tipo de ecuación propuesta con el fin de generar una mayor eficiencia.

- Formular modelos basados en ecuaciones diferenciales, emplear los métodos analíticos y cualitativos más comunes para hallar soluciones e interpretar las mismas.

- Comprender la importancia del cálculo numérico en la solución de problemas, en los que no es posible o es muy difícil hallar soluciones en forma analítica o exacta, reconociendo las limitaciones de las calculadoras y computadoras.

- Conocer y aplicar interpolaciones de funciones conocidas para poder establecer su error de interpolación. También realizar ajustes de datos por mínimos cuadrados y calcular su coeficiente de determinación para verificar la bondad de ajuste en un modelo planteado. 


\section{En la línea de estadística}

Al finalizar los cursos, el estudiante estará preparado para la aplicación y el manejo descriptivo de datos (codificación, tabulación y evaluación de medidas de resumen de datos univariantes y bivariantes). Así mismo, tendrá las habilidades necesarias para el manejo en la evaluación de probabilidades y de los principales modelos de distribución de probabilidad. Además de la estructura lógica deductiva se aportan visiones intuitivas y un sentido de aplicaciones prácticas que le permiten al estudiante aplicar la teoría de la probabilidad y hacer uso de la inferencia y los métodos estadísticos.

\section{Conclusiones}

Se encuentra que en primer semestre en las universidades nacionales algunas empiezan automáticamente la línea del cálculo, mientras que otras ofertan primero un precálculo. En este sentido, la Universidad Distrital Francisco José de Caldas, no ofrece esta oportunidad a los estudiantes. En el primer semestre se evidencia una polisemia en los nombres que contienen las mismas unidades conceptuales, estas se diferencian por la cantidad de créditos de acuerdo con la intensidad horaria. La Universidad Distrital Francisco José de Caldas, oferta un cálculo diferencial, que se articula en unidades conceptuales, intensidad horaria y número de créditos con lo ofertado por las universidades objeto de esta investigación.

La Universidad Distrital Francisco José de Caldas, ha permitido a los docentes de matemáticas, a partir de reflexiones de encuentros, construir un único currículo, representado por los syllabus de los espacios académicos de matemáticas. En este sentido, los beneficios son muchos, por ejemplo, la posibilidad de realizar homologaciones y transferencias a otras instituciones de educación superior, sin ningún inconveniente. Esto fomenta la movilidad y flexibilidad de los estudiantes.

Al realizar el análisis de la información recopilada de los espacios académicos de los planes de estudio de matemáticas, se concluye que existe un buen nivel de uniformidad entre los diferentes programas ofertados por la Universidad Distrital Francisco José de Caldas, que se articula sin ninguna dificultad con las propuestas que tienen otras instituciones de educación superior.

Aunque el tema de deserción y repitencia no es el objeto de este trabajo, y dado el porcentaje tan alto de perdida en las asignaturas de matemáticas, se hace necesario -como lo demuestra esta investigación- repensar la creación de un nuevo espacio académico que contemple la revisión de los conceptos previos al cálculo diferencial y soporte toda la línea del área de las matemáticas.

En la educación superior colombiana el nivel profesional (para ingenierías) debe tener un mínimo de 160 créditos y un máximo recomendado de 180 créditos. Las Universidades que ofertan ingenierías y tecnologías, objeto de este estudio, se encuentran dentro del marco de la ley, la diferencia radica en la distribución de los créditos para cada una de las áreas, incluyendo matemáticas.

Es importante resaltar que el principal beneficiado con el proceso de investigación es la comunidad académica de la universidad Distrital, pues con los resultados obtenidos se pretende replantear los cambios curriculares, de tal forma que promuevan la creación de un nuevo espacio académico que posibilite la adquisición o el perfeccionamiento de los conocimientos previos con los que debe salir cualquier bachiller antes de ingresar a la universidad.

Finalmente, otro aspecto importante y que cabe resaltar es la importancia que otras universidades le han dado al estudio de las matemáticas previas al cálculo. Bajo esta perspectiva, la Universidad Distrital Francisco José de Caldas, podría ofertar cursos de nivelación para los estudiantes que ingresan a la universidad. Esto sería una estrategia que podría mejorar la retención y deserción escolar.

\section{Bibliografía}

Asociación Colombiana de Facultades de Ingeniería-ACOFI. (2005). El impacto de las reformas 
de la educación superior en la formación de ingenieros. Cartagena.

Chaparro, O. (1999). "De la sociedad de la información a la sociedad del conocimiento". En Gómez Buendía, H. (Comp.) ¿Para dónde va Colombia? Santafé de Bogotá: Tercer Mundo y COLCIENCIAS.

Gómez, C. (1990). La Educación Tecnológica en Colombia: análisis de su eficacia externa. En Memorias IV seminario de investigación en educación, Bogotá.

Misas Arango, G. (2004). La educación superior en Colombia: análisis y estrategias para su desarrollo. Bogotá, D. C.: Universidad Nacional de Colombia.

Oviedo, P. (2003). El proceso general de la investigación científica. Guía para la formulación de proyectos de investigación, y cómo definir cada aspecto del proyecto de investigación. Universidad Pedagógica Nacional. Notas de clase Maestría en Educación.
Palacios, M. (2004). La reforma académica que requiere la Universidad Nacional de Colombia. Rectoría Universidad Nacional.

Rico, L. (1998). “Complejidad del currículo de matemáticas como herramienta profesional" [en línea]. En Revista Latinoamericana de investigación en matemática educativa, vol. 1, núm. 001, pp. 22-39. Disponible en http://redalyc. uaemex.mx/pdf/335/33510103.pdf

Universidad Distrital Francisco José de Caldas (2005). Flexibilidad curricular y créditos académicos. Documento de estudio. Vicerrectoría académica.

Universidad Distrital Francisco José de Caldas. (2008). Plan estratégico de Desarrollo 20072016, "Saberes, Conocimientos e Investigación de Alto Impacto para el Desarrollo Humano y Social”. Grupo Editorial Gaia. Bogotá.

Villaveces, J. (2008). “Las competencias matemáticas genéricas de los egresados de la educación superior" [en línea]. Disponible en http:// www.universityofcalifornia.edu/senate/reports/mathcomp.html 